\title{
Características de Composición Corporal, Ángulo de Fase y Agua Corporal en Paracaidistas Chilenos de Elite
}

\author{
Characteristics of Body Composition, Phase Angle and Body Water in Elite Chilean Skydivers
}

\author{
Rodrigo Yáñez-Sepúlveda ${ }^{1,2,3}$; Ildefonso Alvear-Ordenes²; Juan Vargas-Silva ${ }^{4}$; \\ Sam Hernández-Jaña ${ }^{5}$; Jorge Olivares-Arancibia ${ }^{5,6} \&$ Marcelo Tuesta $^{7}$
}

YÁÑEZ-SEPÚLVEDA, R.; ALVEAR-ORDENES, I.; VARGAS-SILVA, J.; HERNÁNDEZ-JAÑA, S.; OLIVARES-ARANCIBIA, J. \& TUESTA, M. Características de composición corporal, ángulo de fase y agua corporal en paracaidistas chilenos de elite. Int. J. Morphol., 39(6):1564-1569, 2021.

RESUMEN: Durante la práctica del paracaidismo, una deficiente composición corporal no sólo puede afectar el rendimiento deportivo, sino que, además, incrementa la probabilidad de sufrir una lesión o accidente grave. Conocer las características de sus componentes, podría ayudar a prevenirlas. El objetivo del estudio fue describir la composición corporal, ángulos de fase y agua corporal total en paracaidistas chilenos de alta competencia. Participaron del estudio 8 paracaidistas profesionales del Team Chile ${ }^{(}(33,4 \pm 4,9$ años $)$ con más de seis años de experiencia. La evaluación de las masas grasa, muscular, libre de grasa, magra y visceral, así como el ángulo de fase y el agua corporal total obtenida a través de impedancia bioeléctrica. Los deportistas presentaron un peso corporal de 76,7 $\pm 5,7 \mathrm{~kg}$, estatura 1,72 $\pm 0,1 \mathrm{~m}$ e IMC 26,0 \pm $1,9 \mathrm{~kg} / \mathrm{m}^{2}$. La composición corporal promedio mostró un 20,6 $\pm 3,0 \%$ de masa grasa, 44,9 $\pm 2,0 \%$ de masa muscular y 79,4 $\pm 3,0 \%$ de masa libre de grasa. El ángulo de fase promedio fue de 7,25 $\pm 0,33^{\circ}$. El agua corporal total de los participantes fue de $44,6 \pm 3,21$. Se concluye que los resultados obtenidos pueden ser utilizados por los profesionales de las ciencias del deporte como valores de referencia para el control de la composición corporal, fase angular y agua corporal en paracaidistas para optimizar el rendimiento deportivo y evitar lesiones.

PALABRAS CLAVE: Impedancia bioeléctrica; Antropometría; Paracaidismo.

\section{INTRODUCCIÓN}

En los últimos años, la popularidad de la práctica del paracaidismo ha incrementado significativamente, sobre todo en los jóvenes (Pillay \& Dada, 2018; Bradke \& Everman, 2020). Este es un deporte de alto riesgo, incluso para la vida, ya que una inadecuada preparación del equipamiento o ejecución de actividades propias de su práctica puede llegar a provocar lesiones musculo esqueléticas graves y, en los casos más complejos, la muerte del deportista (Mohamed et al., 2015; Cavalade et al., 2015; Massimino et al., 2019). Durante el salto, existe una elevada probabilidad de sufrir lesiones traumáticas (Fer et al., 2021), en particular en el momento de la apertura del paracaídas (luxación de hombro) y/o con el contacto con el suelo durante el aterrizaje (fracturas de miembros inferiores) (Westman \& Björnstig,
2007; Nilsson et al., 2013). En todos estos casos, la distribución y características de los componentes de la composición corporal puede incrementar o disminuir las probabilidades de sufrir una lesión. Un ejemplo de esto es el sobrepeso, el cual aumenta el riesgo de lesión de miembros inferiores, sobre todo en la modalidad de tándem, la cual consiste en un salto en paracaídas con dos participantes, utilizando un arnés. En este sentido, la fijación del arnés en las piernas exige que el paracaidista tenga una fuerza efectiva para levantarlas durante el contacto con el suelo, y así evitar lesiones, para esto, será necesaria una preparación física orientada a lograr una composición corporal con un buen desarrollo de la masa y calidad muscular, evitando el aumento de la masa grasa.

\footnotetext{
${ }^{1}$ Escuela de Educación. Pedagogía en Educación Física, Universidad Viña del Mar, Chile.

${ }^{2}$ Laboratorio de Fisiología Aplicada (FISAP). Instituto de Biomedicina (IBIOMED) y Departamento de Ciencias Biomédicas. Universidad de León, España.

${ }^{3}$ Red Iberoamericana de Investigadores en Antropometría Aplicada (RIBA2).

${ }^{4}$ Departamento de Investigación de la Federación Deportiva de la Fuerza Aérea de Chile (FEDEFA).

${ }^{5}$ Grupo IRyS, Escuela de Educación Física, Pontificia Universidad Católica de Valparaíso, Chile.

${ }^{6}$ Grupo AFySE, Investigación en Actividad Física y Salud Escolar, Escuela de Pedagogía en Educación Física, Facultad de Educación, Universidad de las Américas, Santiago, Chile

${ }^{7}$ Exercise and Rehabilitation Sciences Laboratory, School of Physical Therapy, Faculty of Rehabilitation Sciences, Universidad Andres Bello, Santiago 7591538, Chile.
} 
Es reconocido que el entrenamiento en deportistas de elite es capaz de modelar la composición corporal, que será determinante en el rendimiento deportivo de excelencia (Sánchez-Muñoz et al., 2018; Stellingwerff, 2018; Gomez-Ezeiza et al., 2019). En este sentido, las diferentes disciplinas del salto en paracaídas, tales como la formación en caída libre (trabajo relativo secuencial), prueba de estilo, tándem, modalidad de precisión de aterrizaje individual y en equipos, provocaránlas adaptaciones corporales según las necesidades. Incluso en aquellas que son en equipo será necesario en algunos casos mantener características físicas similares. Un ejemplo de esto es la modalidad de formación en caída libre, la cual consiste en formar una figura entre varios participantes afirmados entre sí. Aquí se debe buscar un equilibrio entre el peso, superficie corporal y relación entre las masas muscular y grasa de los participantes. Esto igualará el esfuerzo para mantener la velocidad de caída, reservando energía que permitirá optimizar la formación de figuras en el aire, mejorando los resultados deportivos. Asimismo, un buen desarrollo del componente muscular permitirá mantener la fuerza de sujeción entre los participantes una vez creada la figura. En Chile, el paracaidismo profesional ha alcanzado importantes logros internacionales, entre los cuales se destacan el $25^{\circ}$ lugar obtenido por equipos en el $43^{\circ}$ CISM World Military Parachuting Championship, celebrado el 2019 en la ciudad de Wuhan, República Popular de China. En este mismo evento, uno de los representantes del equipo chileno alcanzó la posición 15 en la modalidad de aterrizaje de precisión. Según la última actualización del ranking mundial por equipos, en Latinoamérica, Chile ocupa el segundo lugar detrás del equipo de Brasil (Fédération Aéronautique Internationale, 2019).

Una de las técnicas de evaluación de la composición corporal más utilizadas en el deporte es la impedancia bioeléctrica (IB) (Di Vincenzo et al., 2019; Campa et al., 2021), la cual permite cuantificar el tejido muscular con un elevado grado de concordancia con el Gold Standard conocido como absorciometría de rayos $\mathrm{X}$ de doble energía o DEXA por su sigla en inglés (Fujimoto et al., 2019). Entre las variables obtenidas por la IB, pero no utilizadas para describir composición corporal se encuentra el ángulo de fase (AF). Este índice se obtiene de la relación entre las medidas de resistencia y reactancia, donde el AF es igual al arco tangente de la reactancia dividido por la resistencia, multiplicado por $180^{\circ} / \pi 1$ (Barbosa-Silva et al., 2005). Sus resultados dependerán de la interacción entre el contenido de agua extracelular e intracelular, la masa celular corporal e integridad celular (Di Vincenzo et al.). La evidencia muestra que una reducción del AF se relaciona con una alteración de la integridad y lesión de la membrana celular, que se ha asociado con un mayor riesgo de lesiones agudas o daño del tejido por sobrecarga (Nescolarde et al., 2013). Por lo tanto, un menor AF se relaciona con una peor calidad muscular (Chen et al., 2020) y contenido de agua corporal intracelular, y viceversa (Denneman et al., 2020). En la actualidad, existe escasa evidencia sobre la composición corporal y calidad muscular de los paracaidistas de alto rendimiento. De acuerdo con el conocimiento de los autores, este es el primer artículo científico que describe detalladamente la condición en la composición corporal y calidad muscular de paracaidistas nacionales de alto rendimiento. En base a lo señalado, el objetivo de nuestro estudio es describir el perfil de composición corporal, fase angular y agua corporal en paracaidistas chilenos de elite durante una competencia.

\section{MATERIAL Y MÉTODO}

Participantes. Muestra intencionada de 8 hombres militares activos (33,4 $\pm 4,9$ años) pertenecientes al equipo nacional de paracaidismo (Team Chile ${ }^{\circledR}$ ) con un promedio de $9,1 \pm 6,3$ años de experiencia profesional y $1198 \pm 776$ saltos efectuados. Cada participante firmó voluntariamente un consentimiento informado escrito, previo a la competencia e inicio de las evaluaciones. En este documento se detallaron los procedimientos de evaluación y el objetivo del estudio. Asimismo, todas las dudas respecto a su participación fueron aclaradas. Esta investigación se llevó a cabo siguiendo las normas éticas establecidas por la declaración de Helsinki para estudios en humanos (World Medical Association, 2013). Además, el estudio fue aprobado por el Comité de Ética Científica (CEC) de la Universidad Viña del Mar (Código 62-19R).

Procedimientos. Este estudio fue desarrollado durante el campeonato nacional de paracaidismo realizado en Santiago de Chile, durante el mes de octubre del año 2018. Los deportistas fueron evaluados durante los primeros 3 días consecutivos a la competición. Se utilizó una tienda militar de campaña habilitada para realizar las evaluaciones de parámetros antropométricos y de IB. Ésta tienda fue ubicada al costado de la pista de aterrizaje (aproximadamente a $50 \mathrm{~m}$ ) y fue acondicionada para mantener un rango de temperatura entre $20^{\circ} \mathrm{C}$ y $22^{\circ} \mathrm{C}$ con calefacción por radiación eléctrica, permitiendo mantener una humedad relativa entre 60 y $70 \%$. Para los días de evaluación, los deportistas debían presentarse sin haber realizado ejercicio intenso, ni consumido alcohol en las 24 horas previas, en ayunas de al menos 4 horas y con posterioridad al vaciado urinario. Las mediciones antropométricas básicas y la IB se llevaron a cabo con los participantes vestidos sólo con ropa interior o pantalón deportivo y sin ningún elemento decorativos (piercing, argollas, etc.), ya fuera metálico o no. Con el sujeto acostado sobre una colchoneta gruesa, en posición de- 
cúbito supino, se procedió a limpiar la piel con alcohol isopropílico de $70^{\circ}$ en la zona de colocación de los electrodos. La colchoneta fue ubicada al menos a 4 metros de algún material metálico u otro conductor de electricidad. Antes de iniciar la medición el sujeto debía estar en reposo durante 5 minutos, tiempo en el cual se revisó que los cables no estuvieran en contacto con el suelo o entrelazados. Después de 2 a 3 minutos, en que los sujetos permanecieron relajados, con los brazos y las piernas levemente abducidos (aproximadamente $40-45^{\circ}$ ), se llevó a cabo la medición por IB.

Recolección de los datos. El peso corporal fue medido con una balanza digital (Ironman, modelo BC-554, Tanita ${ }^{\circledR}$ ), la cual tiene una precisión de $0,1 \mathrm{~kg}$. La estatura se obtuvo utilizando un tallímetro portátil mecánico (modelo 213, SECA $\left.{ }^{\circledR}\right)$, el cual tiene una precisión de $0,5 \mathrm{~cm}$. La composición corporal, AF y agua corporal fueron obtenidos por un impedanciómetrobioeléctrico de multifrecuencias (modelo S10, Inbody $\left.{ }^{\circledR}\right)$. La composición corporal fue obtenida para el cuerpo en su totalidad y algunas zonas corporales separadamente, tales como brazos, piernas y tronco. Aquí se incluyó la masa grasa ( $\mathrm{kg}$ y \%), masa muscular ( $\mathrm{kg}$ y \%), masa libre de grasa $(\mathrm{kg} \mathrm{y} \mathrm{\% )}$, masa magra $(\mathrm{kg})$ y área de grasa visceral $(\mathrm{cm} 2)$. También fueron obtenidos los índices de masa muscular y de masa grasa, los cuales consisten en la normalización de las masas al dividir su valor en kilos por la estatura elevada al cuadrado. Respecto a los volúmenes de agua, se obtuvieron el agua corporal total (1), agua corporal intracelular (1) y agua corporal extracelular (1). La IB fue llevada a cabo con una frecuencia de $50 \mathrm{kHz}$, esta frecuencia presenta mejor relación entre la reactancia y resistencia, permitiendo que la corriente pueda atravesar la membrana celular, generando un análisis preciso a nivel intra y extracelular, permitiendo cuantificar con mayor calidad los tejidos, además se ha visto que las frecuencias superiores a $100 \mathrm{kHz}$ no mejoran la precisión de la estimación de la composición corporal (Marra et al., 2019). Todas las variables de IB fueron calculadas utilizando el programa Lookin Body Software (Inbody®).

Análisis estadístico. Los resultados de las tres evaluaciones para cada variable son presentados en promedios, desviación estándar, mínimo, máximo y coeficiente de variación. Para esto se utilizó el software estadístico SPSS ${ }^{\circledR}$ (versión $25, \mathrm{IBM} \circledast$ ).

\section{RESULTADOS}

En la Tabla I se describen las características antropométricas básicas y composición corporal. El índice de masa corporal (IMC) promedio reveló sobrepeso en los paracaidistas. Además, la masa muscular $(45,0 \pm 2,0 \%, \mathrm{CV}$ : $4,4 \%)$ y la masa libre de grasa $(79,4 \pm 3,0 \%, \mathrm{CV}: 3,8 \%)$ fueron superiores a los valores de referencia para sujetos sanos según sexo y edad. Asimismo, estas últimas son las que presentan un menor porcentaje de variación. Por otro lado, la masa grasa $(15,9 \pm 2,8 \mathrm{~kg}, \mathrm{CV}: 17,9 \%)$ y el área de grasa visceral $(76,2 \pm 11,0 \mathrm{~cm} 2, \mathrm{CV}: 14,4 \%)$ fueron inferiores a los valores de referencia según sexo y edad, pero con mayor porcentaje de variación.

La Tabla II muestra los valores del AF y agua corporal normales. La relación AEC/ACT $(0,4 \pm 0,011$; CV: 1,4 $\%)$ presentó una baja variabilidad, mientras que fue el indi-

Tabla I. Características generales de composición corporal de paracaidistas de alto rendimiento.

\begin{tabular}{lcccc}
\hline Variables & Media \pm DE & Mín & Máx & CV (\%) \\
\hline Peso $(\mathrm{kg})$ & $76,7 \pm 5,7$ & 66,8 & 87,2 & 7,4 \\
Estatura $(\mathrm{m})$ & $1,72 \pm 0,1$ & 1,65 & 1,79 & 2,7 \\
IMC $\left(\mathrm{kg} / \mathrm{m}^{2}\right)$ & $26,0 \pm 1,9$ & 23,7 & 28,5 & 7,4 \\
Masa grasa $(\mathrm{kg})$ & $15,9 \pm 2,8$ & 12,1 & 19,9 & 17,9 \\
Masa grasa $(\%)$ & $20,6 \pm 3,0$ & 16,5 & 25,7 & 14,4 \\
Índice de masagrasa $\left(\mathrm{kg} / \mathrm{m}^{2}\right)$ & $5,4 \pm 0,9$ & 3,9 & 6,7 & 18,0 \\
Masa muscular $(\mathrm{kg})$ & $34,5 \pm 2,8$ & 29,9 & 39,5 & 8,1 \\
Masa muscular $(\%)$ & $45,0 \pm 2,0$ & 41,4 & 47,6 & 4,4 \\
Índice de masa muscular $(\mathrm{kg} / \mathrm{m} 2)$ & $11,7 \pm 0,9$ & 10,6 & 12,9 & 8,0 \\
Masa libre de grasa $(\mathrm{kg})$ & $60,8 \pm 4,4$ & 53,3 & 68,7 & 7,2 \\
Masa libre de grasa $(\%)$ & $79,4 \pm 3,0$ & 74,2 & 83,5 & 3,8 \\
Masa magra brazo derecho $(\mathrm{kg})$ & $3,5 \pm 0,4$ & 2,8 & 4,1 & 10,7 \\
Masa magra brazo izquierdo $(\mathrm{kg})$ & $3,5 \pm 0,4$ & 2,8 & 4,0 & 10,8 \\
Masa magra tronco $(\mathrm{kg})$ & $26,9 \pm 2,1$ & 23,3 & 30 & 7,8 \\
Masa magra pierna derecha $(\mathrm{kg})$ & $9,3 \pm 0,7$ & 8,0 & 10,5 & 7,3 \\
Masa magra pierna izquierda $(\mathrm{kg})$ & $9,2 \pm 0,7$ & 8,0 & 10,3 & 7,0 \\
Área grasa visceral $\left(\mathrm{cm}{ }^{2}\right)$ & $76,2 \pm 11,0$ & 60,90 & 90,5 & 14,4 \\
\hline
\end{tabular}

CV: coeficiente de variación; DE: desviación estándar; IMC: índice de masa corporal; Máx: máximo; Mín: mínimo. 
cador con menor nivel de variación, mientras que las variables con mayor variabilidad fueron el AF pierna derecha $\left(7,1^{\circ} \pm 0,7^{\circ} ; \mathrm{CV}: 10,5 \%\right)$ y el AF pierna izquierda $\left(7,1^{\circ} \pm\right.$ $0,07^{\circ}$; CV: $\left.9,3 \%\right)$.

Tabla II. Ángulo de fase y agua corporal de paracaidistas elite.

\begin{tabular}{lcccc}
\hline Variable & Media y DE & Mín & Máx & CV (\%) \\
\hline AF $\left(^{\circ}\right)$ brazo derecho & $7,0 \pm 0,3$ & 6,5 & 7,3 & 4,1 \\
AF $\left(^{\circ}\right)$ brazo izquierdo & $6,9 \pm 0,3$ & 6,5 & 7,4 & 4,1 \\
AF $\left(^{\circ}\right)$ tronco & $8,2 \pm 0,4$ & 7,8 & 8,9 & 4,8 \\
AF $\left(^{\circ}\right)$ pierna derecha & $7,1 \pm 0,7$ & 5,9 & 7,9 & 10,5 \\
AF $\left(^{\circ}\right)$ pierna izquier da & $7,1 \pm 0,7$ & 6,1 & 7,9 & 9,3 \\
AF $\left(^{\circ}\right)$ total & $7,3 \pm 0,3$ & 6,7 & 7,6 & 4,6 \\
AIC $(1)$ & $28,0 \pm 2,1$ & 24,4 & 31,9 & 7,7 \\
AEC $(1)$ & $16,6 \pm 1,1$ & 14,5 & 18,4 & 6,6 \\
ACT $(1)$ & $44,6 \pm 3,2$ & 39,0 & 50,3 & 7,2 \\
AEC/ACT & $0,4 \pm 0,01$ & 0,4 & 0,4 & 1,4 \\
\hline
\end{tabular}

ACT: agua corporal total: AEC: agua extracelular; AEC/ACT: relación AEC/ACT;AF: ángulo de fase; AIC: agua intracelular; CV: coeficiente de variación. DE: desviación estándar; Máx: máximo; Mín: míDISCUSIÓN nimo.

El presente estudio tuvo por objetivo estudiar la composición corporal, AF y agua corporal en paracaidistas chilenos de alto rendimiento. Todos los resultados obtenidos tuvieron coeficientes de variación menores al $30 \%$, lo cual indica que los promedios son representativos de la muestra estudiada. Al comparar con referencias de hombres sanos, la masa muscular y masa grasa total de los paracaidistas estudiados se encuentran sobre y dentro del rango de normalidad respectivamente (Gallagher et al., 2000; Kuriyan et al., 2008). Al comparar la composición entre ambas piernas y brazos, se observó que el componente de masa magra fue similar, lo cual demostraría un equilibrio bilateral del tejido muscular en los miembros. Ahora bien, a diferencia de la composición corporal, el IMC no es un índice antropométrico adecuado para determinar el efecto del entrenamiento o práctica deportiva sobre la adaptación física y menos si se desea relacionar con el rendimiento deportivo (Gasier et al., 2015; Durán-Agüero et al., 2017). En efecto, en este estudio el IMC promedio de los paracaidistas se ubicó en el rango de sobrepeso (>24,9 kg/m2). Sin embargo, la composición corporal de los sujetos evaluados mostró un nivel de tejidos muscular y graso, adecuados para este tipo de deportistas. Asimismo, el área de la grasa visceral se mantuvo en los rangos normales según predichos (Park et al., 2016). Resultados muy diferentes fueron observados por el grupo de Vázquez-Guzmán et al. (2020) en un grupo de paracaidistas militares mexicanos. En éstos, la masa grasa $(28,1 \%)$ y la masa libre de grasa total $(71,7 \%)$ fueron mayor en $7,5 \%$ y menor en $8,3 \%$ respecto a nuestro estudio, respectivamente. Cabe recordar que la acumulación excesiva de tejido adiposo disminuye el rendimiento físico, ya que, al actuar como lastre, disminuye la eficiencia del esfuerzo realizado. En este sentido, los paracaidistas pueden ver disminuida la posibilidad de generar movimientos que requieren mayor potencia, como por ejemplo durante el aterrizaje a alta velocidad (Westman \& Björnstig). Es así que, se ha podido observar en paracaidistas, que el exceso de grasa corporal también puede alterar la respuesta cognitiva en los momentos de estrés propios de la actividad. Un estudio de Mujica-Parodi et al. (2009) observó una relación elevada entre exceso de grasa corporal con la disminución del rendimiento cognitivo, afectando la precisión de una tarea $(\mathrm{r}=-0,66 ; \mathrm{p}=0,01) \mathrm{y}$ su tiempo de respuesta $(r=-0,62 ; p=0,02)$ durante el salto, disminuyendo el rendimiento deportivo e incrementado el riesgo. Un estudio llevado a cabo en 9 paracaidistas de alto rendimiento, pertenecientes a la fuerza aérea española, mostró promedios de peso $(77,1 \pm 7,4 \mathrm{~kg})$, altura $(1,73 \pm 6,7 \mathrm{~m})$ e IMC $(25,6 \pm 1,9 \mathrm{~kg} / \mathrm{m} 2)$ similares a los registrados en nuestra muestra. Incluso este grupo arrojó un IMC de sobrepeso, tal como ocurrió en nuestro estudio, y el porcentaje de grasa, determinada por IB, fue similar al observado en los deportistas de nuestro estudio, con 20,5 $\pm 3,9 \%$ y 20,6 $\pm 3,0 \%$ respectivamente (Martínez González-Moro et al., 2015). Si bien estos porcentajes se acercan al rango de exceso de adiposidad (Gallagher et al.), Bosy-Westphal et al. (2005) reforzaron la idea de relacionar las masas corporales con el cuadrado de la estatura, sobre todo muscular y grasa. De tal forma es posible comparar las masas de estos tejidos sin ser la altura el factor determinante. A estos cocientes se les denominó índice de masa muscular y masa grasa. Los paracaidistas de nuestro estudio se ubicaron por sobre 3 desviaciones estándar $\left(11,7 \pm 0,9 \mathrm{~kg} / \mathrm{m}^{2}\right)$ y aproximadamente bajo 0,5 desviaciones estándar $\left(5,4 \pm 0,9 \mathrm{~kg} / \mathrm{m}^{2}\right)$ para los índices de masa muscular y grasa corporal promedio, respectivamente. 
Respecto a otros temas relacionados con la medición con IB como el AF y el agua corporal total. Para los paracaidistas evaluados en este estudio, el AF total $\left(7,3 \pm 0,3^{\circ}\right)$ fue similar o levemente superior a lo observado en otros estudios con sujetos sanos no deportistas, según edad y sexo (Kumar et al., 2012; Mattiello et al., 2020), destacándose el AF del tronco como el más alto $\left(8,2 \pm 0,4^{\circ}\right)$. El AF parece ser un indicador más sensible del estado nutricional en comparación con la impedancia, ya que está estrechamente asociado con la integridad celular (Marra et al.). Asimismo, es reconocida su relación positiva con el rendimiento deportivo, ya que estaría asociado con la calidad muscular (Genton et al., 2020). Cabe destacar que la exposición a altas velocidades de caída libre así como la fuerza provocada por la apertura del paracaídas podría incrementar significativamente el esfuerzo físico, dañando sistemáticamente la musculatura entre salto y salto, lo cual podría provocar un desmedro importante del rendimiento durante la competencia e incrementar el riesgo de una lesión. En este sentido, el AF parece ser el indicado para controlar los efectos sobre la salud de los paracaidistas durante el periodo deportivo y la competencia.

Respecto al agua corporal, un estudio llevado a cabo en militares chilenos varones mostró un valor promedio de


gramos, respectivamente (Durán-Agüero et al.). Debido a que el agua tiene una densidad de $1 \mathrm{~kg} / \mathrm{l}$, estos valores son equivalentes a litros de agua corporal. De esta forma, nuestros resultados de ACT AIC y AEC fueron similares a los observados por el grupo de Durán-Agüero et al. para la según edad y sexo. Cabe recordar que cambios bruscos a alta altura desde el vuelo previo al salto provocarán hiperventilación, lo cual favorece la deshidratación corporal. Asimismo, los cambios en la temperatura podrían modificar una redistribución corporal sanguínea, alterando el contenido de agua de los compartimientos corporales, pudiendo afectar en particular de la musculatura esquelética.

En conclusión, la IB permite obtener la composición corporal, ángulo de fase y agua corporal de paracaidistas de alto rendimiento en contexto de una competencia, la cual puede ser utilizada por los profesionales de ciencias del deporte y deportistas para el control y optimización del rendimiento deportivo, así como la prevención de lesiones.

\section{AGRADECIMIENTOS}

A la Fuerza Aérea de Chile (FACH), a la Federación Deportiva de la Fuerza Aérea de Chile (FEDEFA) y especialmente al grupo de presentaciones Boinas Azules por su participación en este estudio. A Camila Flores Encalada, Nutricionista del Centro de Medicina Aeroespacial (CMAE) de la Fuerza Aérea de Chile.

YÁÑEZ-SEPÚLVEDA，R.; ALVEAR-ORDENES，I.; VARGAS-SILVA, J.; HERNÁNDEZ-JAÑA, S.; OLIVARESARANCIBIA, J. \& TUESTA, M. Characteristics of body composition, phase angle and body water in elite Chilean skydivers. Int. J. Morphol., 39(6):1564-1569, 2021.

SUMMARY: Unsuitable body composition in skydivers not only affect the sport performance but also could increase the probability of risk injury or severe accident; hence, to determine body composition characteristics could be helpful to prevent such events. This study aimed to describe body composition, phase angle, and total body water in highly trained Chilean skydivers. Eight Team Chile ${ }^{\circledR}$ professional skydivers $(33.4 \pm 4.9$ years $)$ with more than 6 years of experience participated in this study. Fat mass, muscle mass, fat-free mass, lean mass, visceral mass, phase angle, and total body water were measured by bioelectrical impedance. Skydivers had a bodyweight of $76.7 \pm 5.7 \mathrm{~kg}$, a height of $1.72 \pm 0.1$ $\mathrm{m}$, and a body mass index of $26.0 \pm 1.9 \mathrm{~kg} / \mathrm{m} 2$. Regarding body composition, they had a fat mass of $20.6 \pm 3.0 \%$, a muscle mass of $44.9 \pm 2.0 \%$, and a fat-free mass of 79.4 $\pm 3.0 \%$. Phase angle and total body water were $7.25 \pm 0.3^{\circ}$ and $344.6 \pm 3.2$, respectively. The present findings could be useful to science sport professionals as reference values of body composition, phase angle, and total body water of highly trained skydivers to improve sports performance and avoid injuries.

KEY WORDS: Bioelectrical impedance; Anthropometry; Skydiving.

\section{REFERENCIAS BIBLIOGRÁFICAS}

Barbosa-Silva, M. C.; Barros, A. J.; Wang, J.; Heymsfield, S. B.; \& Pierson Jr., R. N. Bioelectrical impedance analysis: population reference values for phase angle by age and sex. Am. J. Clin. Nutr., 82(1):49-52, 2005.

Bosy-Westphal, A.; Danielzik, S.; Dörhöfer, R. P.; Later, W.; Wiese, S. \& Müller, M. J. Phase angle from bioelectrical impedance analysis: population reference values by age, sex, and body mass index. JPENJ. Parenter. Enteral Nutr., 30(4):309-16, 2006.

Bradke, B. S. \& Everman, B. R. Mild hypoxia of a skydiver making repeated, medium-altitude aircraft exits. Aerosp. Med. Hum. Perf., 91(2):110-5, 2020

Campa, F.; Toselli, S.; Mazzilli, M.; Gobbo, L. A. \& Coratella, G. Assessment of body composition in athletes: a narrative review of available methods with special reference to quantitative and qualitative bioimpedance analysis. Nutrients, 13(5):1620, 2021.

Cavalade, M.; Papadopoulou, V.; Theunissen, S. \& Balestra, C. Heart rate variability and critical flicker fusion frequency changes during and after parachute jumping in experienced skydivers. Eur. J. Appl. Physiol., 115(7):1533-45, 2015.

Chen, J.; Lu, K.; Chen, H.; Hu, N.; Chen, J.; Liang, X.; Qin, J. \& Huang, W. Trunk skeletal muscle mass and phase angle measured by bioelectrical impedance analysis are associated with the chance of femoral neck fracture in very elderly people. Clin. Interv. Aging, 15:88995,2020 . 
Denneman, N.; Hessels, L.; Broens, B.; Gjaltema, J.; Stapel, S.; Stohlmann, J.; Nijsten, M. \& Oudemans-van Straaten, H. Fluid balance and phase angle as assessed by bioelectrical impedance analysis in critically ill patients: a multicenter prospective cohort study. Eur. J. Clin. Nutr., 74(10):1410-9, 2020.

Di Vincenzo, O.; Marra, M. \& Scalfi, L. Bioelectrical impedance phase angle in sport: A systematic review. J. Int. Soc. Sports Nutr., 16:49, 2019.

Durán-Agüero, S.; Maraboli Ulloa, D.; Fernández-Frías, F. \& CubillosSchmied, G. Composición corporal en soldados chilenos del Regimiento Buín. Rev. Esp. Nutr. Hum. Diet., 21(1):11-7, 2017.

Fédération Aéronautique Internationale. 43rd Cism World Military Parachuting Championship 2019. Wuhan City, Fédération Aéronautique Internationale, 2019. Disponible en: http:// results.worldskydiving.org/FrontEnd/CompetitionCollection/1033

Fer, C.; Guiavarch, M.; \& Edouard, P. Epidemiology of skydiving-related deaths and injuries: A 10-years prospective study of 6.2 million jumps between 2010 and 2019 in France. J. Sci. Med. Sport, 24(5):448-53, 2021

Fujimoto, K.; Inage, K.; Eguchi, Y.; Orita, S.; Toyoguchi, T.; Yamauchi, K.; Suzuki, M.; Kubota, G.; Sainoh, T.; Sato, J.; et al. Dual-energy $\mathrm{x}$-ray absorptiometry and bioelectrical impedance analysis are beneficial tools for measuring the trunk muscle mass of patients with low back pain. Spine Surg. Relat. Res., 3(4):335-41, 2019.

Gallagher, D.; Heymsfield, S.; Heo, M.; Jebb, S.; Murgatroyd, P. \& Sakamoto, Y. Healthy percentage body fat ranges: an approach for developing guidelines based on body mass index. Am. J. Clin. Nutr., 72(3):694-701, 2000.

Gasier, H. G.; Hughes, L. M.; Young, C. R. \& Richardson, A. M. Comparison of body composition assessed by Dual-Energy X-Ray absorptiometry and BMI in current and former U.S. navy service members. Plos One., 10(7): 0132157, 2015.

Genton, L.; Mareschal, J.; Norman, K.; Karsegard, V. L.; Delsoglio, M.; Pichard, C.; Graf, C. \& Herrmann, F. R. Association of phase angle and running performance. Clin. Nutr. ESPEN, 37:65-8, 2020.

Gomez-Ezeiza, J.; Tam, N.; Torres-Unda, J.; Granados, C. \& SantosConcejero, J. Anthropometric characteristics of top-class Olympic race walkers. J. Sports Med. Phys. Fitness, 59(3):429-33, 2019.

Kumar, S.; Dutt, A.; Hemraj, S.; Bhat, S. \& Manipadybhima, B. Phase angle measurement in healthy human subjects through bio-impedance analysis. Iran. J. Basic Med. Sci., 15(6):1180-4, 2012.

Kuriyan, R.; Thomas, T. \& Kurpad, A. Total body muscle mass estimation from bioelectrical impedance analysis \& simple anthropometric measurements in Indian men. Indian J. Med. Res., 127(5):441-6, 2008.

Marra, M.; Sammarco, R.; De Lorenzo, A.; Iellamo, F.; Siervo, M.; Pietrobelli, A.; Donini, L. M.; Santarpia, L.; Cataldi, M.; Pasanisi, F.; et al. Assessment of body composition in health and disease using bioelectrical Impedance Analysis (BIA) and Dual Energy X-Ray Absorptiometry (DXA): a critical overview. Contrast Media Mol. Imaging, 2019:3548284, 2019.

Martínez González-Moro, I.; Mesas Quesada, C.; Galián Nicolás, E. \& Lomas Albaladejo, J. Evaluation of the corporal composition of professional acrobatic parachutists with bioelectrical impedance and anthropometry. Nutr. Hosp., 32 Suppl. 2:10315, 2015.

Massimino, S.; Rinella, S.; Buscemi, A.; Similia, E.; Perciavalle, V.; Perciavalle, V.; Petralia, M. C.; Di Corrado, D.; Laspina, A. \& Coco, M. Digit ratio, personality and emotions in skydivers. Biomed. Rep., 10(1):39-46, 2019.

Mattiello, R.; Amaral, M A.; Mundstock, E. \& Ziegelmann, PK. Reference values for the phase angle of the electrical bioimpedance: Systematic review and meta-analysis involving more than 250,000 subjects. Clin. Nutr., 39(5):1411-7, 2020.

Mohamed, S.; Favrod, V.; Philippe, R. A. \& Hauw, D. The situated management of safety during risky sport: learning from skydivers' courses of experience. J. Sports Sci. Med., 14(2):340-6, 2015.
Mujica-Parodi, L.; Renelique, R. \& Taylor, M. K. Higher body fat percentage is associated with increased cortisol reactivity and impaired cognitive resilience in response to acute emotional stress. Int. J. Obes. (Lond.), 33(1):157-65, 2009.

Nescolarde, L.; Yanguas, J.; Lukaski, H.; Alomar, X.; Rosell-Ferrer, J. \& Rodas, G. Localized bioimpedance to assess muscle injury. Physiol. Meas., 34(2):237-45, 2013.

Nilsson, J.; Fridén, C.; Burén, V.; Westman, A.; Lindholm, P. \& Ang, B. O. Musculoskeletal pain and related risks in skydivers: a populationbased survey. Aviat. Space Environ. Med., 84(10):1034-40, 2013.

Park, K.; Lee, D.; Lee, J.; Kim, Y.; Jung, K.; Kim, K.; Kwak, S.; Choi, S.; Park, K.; Jang, H.; et al. Comparison between two methods of bioelectrical impedance analyses for accuracy in measuring abdominal visceral fat area. J. Diabetes Complications, 30(2):343-9, 2016.

Pillay, D. \& Dada, S. Skydiving: The audiological perspective. S. Afr. J. Commun. Disord., 65(1):1-4, 2018.

Sánchez-Muñoz, C.; Muros, J. J. \& Zabala, M. World and Olympic mountain bike champions' anthropometry, body composition and somatotype. J. Sports Med. Phys. Fitness, 58(6):843-51, 2018.

Stellingwerff, T. Case study: body composition periodization in an olympic-level female middle-distance runner over a 9-year career. Int. J. Sport Nutr. Exerc. Metab., 28(4):428-33, 2018.

Vázquez-Guzmán, M.; Carrera-Rodríguez, G.; Durán-García, A. \& Gómez-Ortiz, O. Correlación del índice de masa corporal con el índice de masa grasa para diagnosticar sobrepeso y obesidad en población militar. Rev. Sanid. Mil., 70:505-15, 2020.

Westman, A. \& Björnstig, U. Injuries in Swedish skydiving. Br. J. Sports Med., 41(6):356-64, 2007.

World Medical Association. World Medical Association Declaration of Helsinki: Ethical principles for medical research involving human subjects. JAMA, 310(20):2191-4, 2013.

\author{
Dirección para correspondencia: \\ Rodrigo Yáñez-Sepúlveda \\ Escuela de Educación \\ Pedagogía en Educación Física \\ Campus Rodelillo \\ Agua Santa 705 \\ Viña del Mar \\ CHILE
}

\section{E-mail: rodrigo.yanez@uvm.cl}

Recibido : 05-08-2021

Aceptado: 30-08-2021 